Research Article

\title{
How to Mitigate Theme Park Crowding? A Prospective Coordination Approach
}

\author{
Yuguo Yuan ${ }^{1}$ and Weimin Zheng $\mathbb{D}^{2}$ \\ ${ }^{1}$ Chengyi University College, Jimei University, Xiamen 361021, China \\ ${ }^{2}$ School of Management, Xiamen University, Xiamen 361005, China \\ Correspondence should be addressed to Weimin Zheng; zhengweimin@xmu.edu.cn
}

Received 16 June 2017; Revised 7 January 2018; Accepted 8 March 2018; Published 15 April 2018

Academic Editor: Carmen Castillo

Copyright (c) 2018 Yuguo Yuan and Weimin Zheng. This is an open access article distributed under the Creative Commons Attribution License, which permits unrestricted use, distribution, and reproduction in any medium, provided the original work is properly cited.

\begin{abstract}
Crowding is a key factor in tourists' experience in theme parks, and mitigating crowding makes parks more competitive. This study examines how to effectively mitigate crowding in theme parks. First, a Markov-based method is developed to predict the spatial-temporal distribution of tourists in the park. Then, a prospective coordination approach based on the tourist distribution prediction is proposed. To evaluate the performance of this approach, an experiment is constructed using an agent-based simulation platform. The results indicate that the proposed method significantly outperforms existing methods. Furthermore, we conduct two experiments and, based on the results, offer several recommendations for crowd management.
\end{abstract}

\section{Introduction}

Theme parks are important tourism products that engage visitors' imagination and create enjoyable experiences for tourists [1]. After several decades of steady growth, the theme park market is slowing in terms of new developments and faces competition from other leisure and tourism products $[2,3]$. To survive in an increasingly competitive marketplace, theme parks need to improve tourists' experience and make parks more competitive [4]. Crowding is a common experience in theme parks, a major contributor to tourists' dissatisfaction [5-9], and a significant determining factor in their intention to attend $[8,10]$. Therefore, crowding management is fundamental to improving tourists' experience and making theme parks more competitive [11].

Several factors may contribute to crowding in theme parks. For example, when the demand exceeds the carrying capacity, some necessary measures should be taken to balance supply and demand [12]. However, crowding in theme parks is often more complex than these overall numbers suggest, as tourists distribute themselves unevenly throughout parks $[5,13,14]$. Therefore, a key to addressing crowding lies in the control of tourists' visit sequences, especially in situations where tourists are somewhat flexible in their itineraries [5].
In recent decades, academics and policymakers have become increasingly interested in methods for managing the local crowding that results from the uneven distribution of tourists. We have analyzed the current coordination approaches and identified a range of methods for improving crowding mitigation. Most of these methods coordinate tourists' visit sequences based on the current crowd situation, which leads to oscillating crowds due to the delay between the decision making and effect emergence $[15,16]$. Some studies have attempted to control crowding oscillations using estimates of future crowding $[17,18]$. However, these studies have considered only one service facility; controlling the oscillations and estimating future crowds are much more difficult in theme parks, which contain numerous interconnected rides [16].

This study proposes a Markov-based method to predict the spatial-temporal distribution of tourists in the park and then develops a prospective coordination approach based on the predicted distribution of tourists. To evaluate the performance of this approach, an experiment is constructed using an agent-based simulation platform. The results indicate that the proposed method significantly outperforms existing methods. Furthermore, we conduct two experiments 
and, based on the results, offer several recommendations for crowd management.

This study contributes to the literature on tourism management by developing a tourist distribution prediction method that achieves a beneficial trade-off between prediction accuracy and efficiency and a prospective coordination approach to mitigate crowding. This new approach avoids the crowding oscillation problem and achieves better performance than existing methods. Our approach may be of particular interest to the tourism sector. The more reasonable coordination mechanisms that we recommend will improve tourists' experience by reducing their unvalued time. The approach may also benefit theme parks, as it provides an improved means for crowd mitigation, thus making parks more competitive.

The remainder of this paper is organized as follows. Section 2 gives an overview of the related research on crowding mitigation and predicting tourist distribution. In Section 3, we present several definitions and our methodological framework, after which the prospective coordination approach is described in detail. Section 4 provides the experimental results and an evaluation of the performance of the approach. We conclude the paper in Section 5 and propose a possible direction for future research.

\section{Literature Review}

2.1. Crowding Mitigation. In recreational contexts, crowding is the state in which the utility of a tourist diminishes or his/her costs for doing an activity increase due to the presence of other tourists [19]. Previous research on crowding can be categorized into two main streams. The first stream focuses on defining crowding and perceptions of crowding [20,21] and on the social and ecological effects of crowding [22-25]. The other stream of research focuses on crowding mitigation $[5,26]$. Our study is part of the second stream of research and is specifically interested in exploring methods for crowding mitigation in theme parks.

Crowding is much more common in theme parks and is associated with much longer waiting time than in other tourist attractions [9]. Many studies have shown that crowding is often worse than it needs to be due to the uneven distribution of tourists in a park $[5,13,14]$. Therefore, a key to addressing crowding in parks lies in coordinating tourists' visit sequences, especially when tourists are somewhat flexible in their itineraries [5]. The practice of directing variable time-related demand in a closed system is sometimes referred to as "load balancing" or "scheduling," terms originating in mechanical engineering and production scheduling [27] that have since expanded to include leisure-demand management $[5,28]$.

In recent years, academics and practitioners have been increasingly involved in studying coordination control. Kawamura et al. [15] proposed a coordination approach based on the current crowding situation of each ride in a park. Ge et al. [29] put forward a concept of regional spacetime load (RSTL), which took the spatial structure, carrying capacity, and duration time of rides into consideration when evaluating the crowding situation, and then coordinated the tourists' tour routes based on the RSTL. Zheng et al. [30] evaluated the performance of three coordination approaches based on a multiagent simulation and believed that both the crowding situation and distance between each pair of rides should be taken into account when coordinating tourists' itineraries. In addition to crowding situations and distance, some studies have considered tourists' preferences [31, 32]. Ahmadi [26] coordinated tourists' routing sequences by optimizing a ride's capacity and then generating the desired transition pattern.

The coordination control approaches proposed in these studies are formulated according to the current situation and ignore future projections. This creates oscillations in crowding due to the delay between the decision making and effect emergence $[15,16]$. To solve the delay problem, tourists should be provided with global information about the future crowding situation rather than the current crowding state. In practice, the most successful and widely adopted approaches to estimating the future crowding situation in theme parks have been based on the concept of reservation [5]. In these approaches, tourists are encouraged to register their next destination into a system, which enables the system to more accurately estimate the future crowding situation and to moderate the oscillations in queue lengths $[5,16]$. However, having to continually register their next destination is a nuisance to tourists. To compensate them for their efforts, priority queues may be made available to tourists who are willing to register their next destination. However, priority lines may lead to perceptions of unfairness [33]. Therefore, a more effective prospective coordination approach for crowding mitigation is becoming imperative.

2.2. Tourists Distribution Prediction. Tourism demand modelling and forecasting in a capacity-constrained service industry has been a major research problem, and these issues play a fundamental role in the administration and planning of destinations [34]. A number of articles on tourism demand forecasting have been published over the last decade, and a multitude of methods have been proposed. Good overviews are provided in the literature reviews of S. F. Witt and C. A. Witt [35], Lim [36], Li et al. [37], and Song and Li [38]. Most of these studies examined demand forecasting for a single object (e.g., tourist destination, tourist attraction) and ignored the relationships between different objects, leading to poor prediction performance in complex situations [39].

More recent studies have tended to explore demand in situations with multiple objects with spatial relationships or, in other words, have adopted the concept of tourist distribution [40]. Zhang et al. [41] investigated the spatial dependence and mechanisms of international and domestic tourist distributions in 299 cities in China using a set of GIS-based spatial statistical tools. Yang and Wong [42] applied exploratory spatial data analysis to investigate the spatial distribution of inbound and domestic tourist flows to cities in China and their growth rates. Yang and Wang [43] provided insights into inbound tourism flow patterns in China's cities using exploratory space-time analysis. These studies revealed the relationships between spatial structure, tourist movement/flow, and tourists distribution at the 


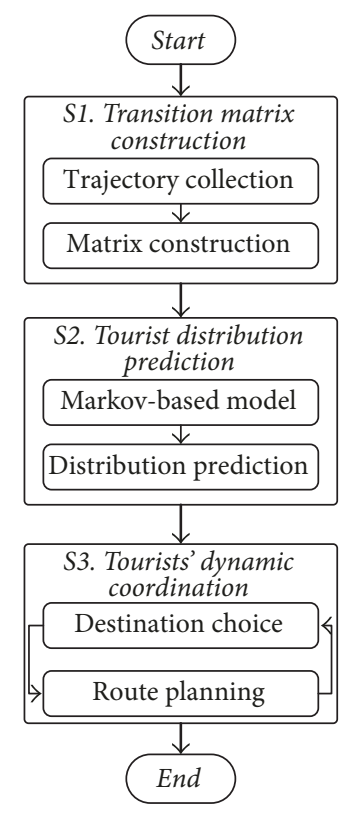

FIGURE 1: Methodological framework.

macroscopic scale and broadened perspectives on tourism demand. However, to date, few studies have attempted to predict tourist distribution within a tourist attraction. A tourist attraction can be regarded as a relatively closed system [44], and intra-attraction tourist movements are complicated and interactive [45, 46]. Zheng et al. [45] proposed a heuristic method to predict a tourist's next location and then predicted the spatial distribution of all of the tourists within the attraction according to the potential location of an individual. Ren et al. [47] predicted tourist distribution using an improved $k$-means method. To our knowledge, these two studies have been the best attempts to predict intraattraction tourist distribution. These pioneering methods are time consuming, and finding a trade-off between accuracy and efficiency in tourist distribution prediction is becoming increasingly important as interest grows in methods for realtime crowding mitigation.

\section{Methodologies}

3.1. Definition and Framework. In this section, we first clarify some terms and variables used in this study and then introduce the framework of our prospective coordination approach.

(1) Definition. For clarity, Abbreviations lists the mathematical notations used in this study and their meanings.

(2) Framework. The methodology has three steps: constructing a transition matrix, predicting tourist distribution, and coordinating tourists' movements. The overall framework is shown in Figure 1.

3.2. Step 1: Transition Matrix Construction. Most theme parks contain numerous interconnected rides [13], which can be abstracted as a network: $G=(V, E, W)$, where $V=$ $\left\{v_{1}, v_{2}, \ldots, v_{N}\right\}$ is the set of rides, $E=\left\{\left(v_{i}, v_{j}\right) \mid v_{i}, v_{j} \in V, v_{i} \neq\right.$ $\left.v_{j}\right\}$ represents the edges between the rides, and $W=\left\{w_{i j} \mid\right.$ $\left.\left(v_{i}, v_{j}\right) \in E\right\}$ denotes the travel time between each pair of rides.

If the $k$ th tourist is at ride $v_{i}$ at moment $t$, set 0 - 1 discrete variable $x_{i k}(t)=1$; otherwise $x_{i k}(t)=0$. Then the number of tourists at ride $v_{i}$ at moment $t$ can be calculated according to (1), where $Q$ denotes the total number of tourists:

$$
\phi_{i}(t)=\sum_{k=1}^{Q} x_{i k}(t) .
$$

If the $k$ th tourist visits ride $v_{j}$ right after visiting $v_{i}$ at moment $t$, set 0 -1 discrete variable $y_{i j k}(t)=1$; otherwise $y_{i j k}(t)=0$. The transition probability from $v_{i}$ to $v_{j}$ at moment $t$ can then be calculated using (2), and the spatial-temporal transition probability matrix at moment $t$ is denoted as $\mathbf{P}(t)$, as shown in (3):

$$
\begin{aligned}
\delta_{i j}(t) & =\frac{\sum_{k=1}^{Q} y_{i j k}(t)}{\phi_{i}(t)}, \quad 0 \leq \delta_{i j}(t) \leq 1, \quad \sum_{j=1}^{N} \delta_{i j}(t)=1, \\
\mathbf{P}(t) & =\left[\begin{array}{cccc}
\delta_{11}(t) & \delta_{12}(t) & \cdots & \delta_{1 N}(t) \\
\delta_{21}(t) & \delta_{22}(t) & \cdots & \delta_{2 N}(t) \\
\vdots & \vdots & \ddots & \vdots \\
\delta_{N 1}(t) & \delta_{N 2}(t) & \cdots & \delta_{N N}(t)
\end{array}\right]
\end{aligned}
$$

3.3. Step 2: Tourist Distribution Prediction. The number of tourists at ride $v_{i}$ at moment $t$ is denoted as $\phi_{i}(t)$ (shown in Section 3.2), so the tourist distribution at moment $t$ can be represented as $D V(t)=\left|\phi_{1}(t), \phi_{2}(t), \ldots, \phi_{N}(t)\right|$. Let $\pi_{i}(t)$ be the proportion of $\phi_{i}(t)$ in the total number of tourists $(Q)$, as shown in

$$
\pi_{i}(t)=\frac{\phi_{i}(t)}{Q}, \quad\left(0 \leq \pi_{i}(t) \leq 1\right) .
$$

If the tourists' distribution at any given moment is considered a state, then the states at moment $t$ can be denoted as (5), and the set of states is $\mathbf{S}(t)=[\mathbf{S}(0), \mathbf{S}(1), \ldots, \mathbf{S}(T)]$. Accordingly, we propose a Markov-based model to predict the tourist distribution for the next moment, on the assumption of first-order dependence [48]; that is, the probability distribution of the next state is independent of past states (as shown in (6)). According to the Markov property, the state of moment $\left(t_{0}+\Delta t\right)$ can be predicted by $(7)$ :

$$
\begin{aligned}
& \mathbf{S}(t)=\left[\pi_{1}(t), \pi_{2}(t), \pi_{3}(t), \ldots, \pi_{N}(t)\right], \\
& \mathbf{S}(t+1)=\mathbf{S}(t) \times \mathbf{P}(t) \\
& =\left[\pi_{1}(t), \pi_{2}(t), \ldots, \pi_{N}(t)\right] \\
& \times\left[\begin{array}{cccc}
\delta_{11}(t) & \delta_{12}(t) & \cdots & \delta_{1 N}(t) \\
\delta_{21}(t) & \delta_{22}(t) & \cdots & \delta_{2 N}(t) \\
\vdots & \vdots & \ddots & \vdots \\
\delta_{N 1}(t) & \delta_{N 2}(t) & \cdots & \delta_{N N}(t)
\end{array}\right],
\end{aligned}
$$




$$
\begin{aligned}
\mathbf{S} & \left(t_{0}+\Delta t\right) \\
& =\left[\pi_{1}\left(t_{0}+\Delta t\right), \pi_{2}\left(t_{0}+\Delta t\right), \ldots, \pi_{N}\left(t_{0}+\Delta t\right)\right] \\
& =\mathbf{S}\left(t_{0}\right) \times \prod_{t}^{t_{0}+\Delta t} \mathbf{P}(t) \\
& =\left[\pi_{1}\left(t_{0}\right), \pi_{2}\left(t_{0}\right), \ldots, \pi_{N}\left(t_{0}\right)\right] \times \prod_{t}^{t_{0}+\Delta t} \mathbf{P}(t) .
\end{aligned}
$$

Finally, the number of tourists at ride $v_{i}$ at moment $\left(t_{0}+\right.$ $\Delta t)$ can be calculated using (8), where $\tilde{\pi}_{i}\left(t_{0}+\Delta t\right)$ is the predicted proportion of tourists at ride $v_{i}$ at moment $\left(t_{0}+\Delta t\right)$ :

$$
\widetilde{\phi}_{i}\left(t_{0}+\Delta t\right)=\tilde{\pi}_{i}\left(t_{0}+\Delta t\right) \cdot Q \text {. }
$$

3.4. Step 3: Tourists' Dynamic Coordination. Organizers of large-scale exhibitions use two methods of tourist coordination control: (1) designing an overall route for tourists based on their preferences and group coordination or (2) deciding the next visiting stop for tourists based on a preferential selection mechanism [31]. Due to the complexity and interactivity of tourist movements in a theme park, the latter coordination control methods have shown greater promise for crowding mitigation in theme parks [31].

Most existing coordination mechanisms organize tourists' visit sequences based on the current crowd situation. This leads to oscillating crowds, due to the delay between decision making and effect emergence $[15,16]$. In this section, we seek to fill this gap by proposing a dynamic coordination mechanism that would organize visitors' tour routes based on a predicted distribution of tourists throughout the park, as illustrated in Section 3.3. Specifically, we calculate the potential cost (PC) for each candidate ride in the CRS. The candidate ride $v_{i}$ with the lowest potential cost is selected as the next destination. The potential cost (PC) includes travel time between the current ride and the candidate ride $v_{i}\left(w_{o i}\right)$, in addition to the potential waiting time at $v_{i}$ when the tourist arrives at this ride; potential waiting time relates to the number of tourists at the candidate ride at that time. We describe how to determine the next destination based on definitions outlined in the pseudocode shown in Algorithm 1. Given a candidate ride set (CRS), distance matrix (DM), and a predicted tourist distribution $(\overline{\mathrm{DV}})$, obtained according to the methods given in Section 3.3, the output of our algorithm is the optimal next destination (lines (1)-(2) in Algorithm 1). The whole process of coordination is described in lines (3)-(11) in Algorithm 1. If there are any candidate rides in CRS (line (3) in Algorithm 1), then the potential cost (PC) for each ride in the CRS is calculated based on (9) (lines (4)-(6) in Algorithm 1), where $w_{o i}$ refers to the travel time between the current ride and the candidate ride $v_{i}$ and can be obtained based on distance matrix (DM), while $\tilde{\pi}_{i}\left(t+w_{o i}\right) \cdot Q$ and $\tilde{\pi}_{i}\left(t+w_{o i}\right) \cdot Q \cdot t_{i} / c_{i}$ represent the predicted number of tourists and the potential waiting time when the focal tourist arrives at $v_{i}$, respectively. $v_{i}$ with the lowest potential cost is selected as the next destination and is removed from the CRS (see lines (8)-(9) in Algorithm 1).

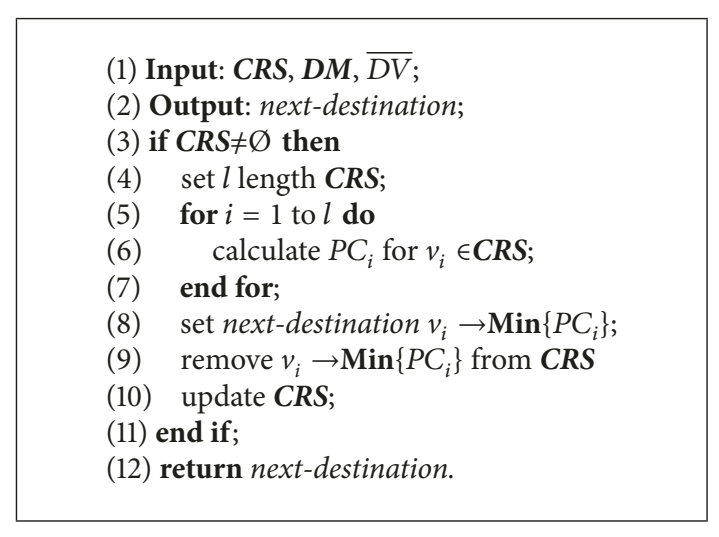

Algorithm 1: Dynamic coordination mechanism.

The CRS is updated and the process stops until there are no longer candidate rides in $\mathrm{CRS}(\mathrm{CRS}=\varnothing)$.

$$
\mathrm{PC}_{i}=w_{o i}+\frac{\tilde{\pi}_{i}\left(t+w_{o i}\right) \cdot \mathrm{Q}}{c_{i}} \cdot t_{i}
$$

\section{Performance Evaluation and Discussion}

To evaluate the performance of our proposed prospective coordination approach, we design simulation experiments based on an agent-based simulation platform. Recently, agent-based simulations have been used to study the complex phenomenon of tourism areas [49-51], as they allow decision makers to evaluate changes without going through the potentially traumatic experience of changing procedures before the results are known [52].

4.1. Simulation Experiment Design. The sample theme park illustrated in Figure 2 is used to test the feasibility of the proposed prospective coordination approach. The theme park includes five theme regions, twenty-five recreation rides, and one entrance/exit. Its operating hours are from 8 a.m. to $10: 00$ p.m. The basic information for each ride is shown in Table 1 , where $t_{i}$ and $c_{i}$ denote the duration and carrying capacity of ride $v_{i}$, respectively.

Our simulation experiment includes the following three steps.

(1) Spatial Trajectories Generation. Tsai and Chung [13] summarized the characteristics of tourists' tour routes in theme parks. In this study, we simulate 2,000 tourists' spatial trajectories based on these characteristics and delete the trajectories that are followed by fewer than five tourists, as the lack of representativeness. The remaining 1,872 trajectories are incorporated into 50 different trajectories, as shown in Table 2, where $t_{n}$ denotes the number of tourists who visit the park along their corresponding trajectories, and the sum of $t_{n}$ is equal to 1,872 .

(2) Spatial-Temporal Trajectories Generation. Time is an important factor in the prediction of tourist movements and crowding mitigation [53]. However, the spatial trajectories 


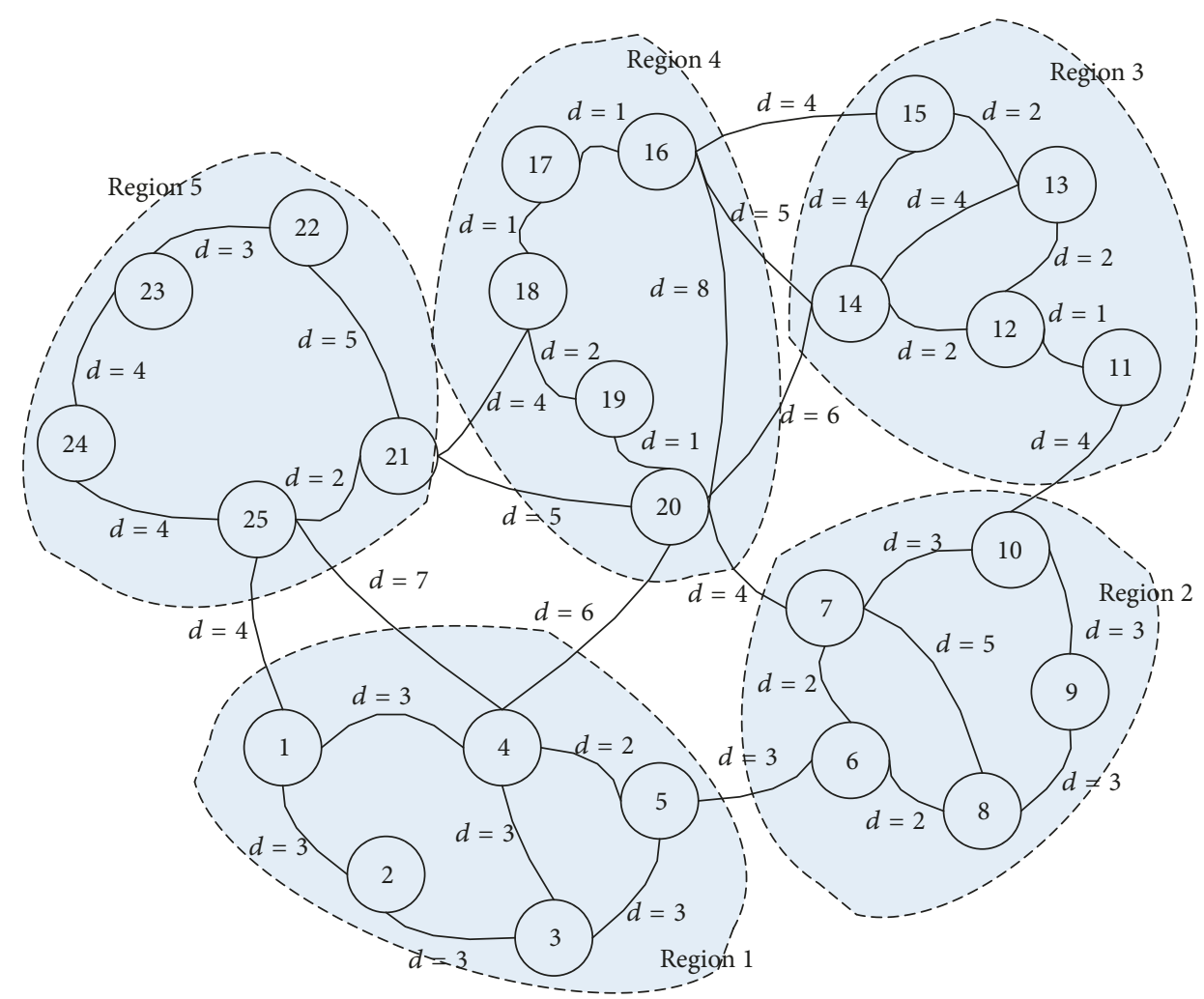

FIGURE 2: Layout of the sample theme park.

TABLE 1: Basic ride information.

\begin{tabular}{lcc}
\hline Ride & $t_{i}$ & $c_{i}$ \\
\hline$v_{1}$ & 2 & 80 \\
$v_{2}$ & 3 & 150 \\
$v_{3}$ & 5 & 140 \\
$\vdots$ & $\vdots$ & $\vdots$ \\
$v_{24}$ & 4 & 200 \\
$v_{25}$ & 6 & 300 \\
\hline
\end{tabular}

listed in Table 2 do not incorporate a timestamp. Therefore, based on the spatial trajectories and $t n$, we simulate 10,000 tourists for each day. The tourists enter the park between 8 a.m. and 10 a.m., and their arrival distribution (the number of tourists arriving at the park every minute) follows the Poisson distribution, a discrete probability distribution that denotes the probability of a given number of events occurring in a fixed interval of time, given that the events occur with a known constant rate and independently of the time since the last event [54]. We repeat the simulation for 100 days, obtaining 10,000 spatial-temporal trajectories for each day.

(3) Spatial-Temporal Transition Matrix Construction. For simplicity, we construct a spatial-temporal transition matrix $\left(\mathrm{ST}^{2} \mathrm{M}\right)$ using 30-minute time intervals rather than 1-minute intervals. The operating hours are divided into 2830 -minute time intervals, such as 8:00-8:30 and 8:30-9:00. After combining the 10,000 spatial-temporal trajectories for each day with the time intervals, $28 \mathrm{ST}^{2} \mathrm{M}$ are obtained. In addition, we average the data for 100 days to minimize errors. Finally, the spatial-temporal transition matrix of each time interval is obtained, which is used to predict tourist distribution.

4.2. Tourist Distribution Prediction. Unlike the tourist distribution prediction approaches proposed by Zheng et al. [45] and Ren et al. [47], we construct a spatial-temporal transition matrix for each time interval based on the travel experience of historical tourists (as shown in (6)). We then predict the overall tourist distribution and the number of tourists at each ride for each moment using (7) and (8), respectively. To evaluate the performance of tourist distribution prediction, we illustrate the process by taking $v_{6}$ as an example: the actual values of tourist numbers at $v_{6}$ for each minute are recorded and shown as red lines in Figure 3, while the blue lines in Figure 3 denote the value predicted by our method.

4.3. Performance Evaluation. To evaluate the performance of our proposed approach, we first construct an evaluation indicator. Most of the tourists in theme parks seek to minimize waiting time and travel times between rides and to spend as much time as possible engaged in activities [13]. Therefore, in this study, we adopt the proportion of unvalued time (NVT\%) as the evaluation indicator.

If the $k$ th tourist is waiting at ride $v_{i}$ at moment $t$, set $0-1$ discrete variable $w t_{i k}(t)=1$; otherwise $w t_{i k}(t)=0$. If the $k$ th tourist visits $v_{j}$ right after $v_{i}$, set 0 - 1 discrete variable $z_{i j k}=1$; otherwise $z_{i j k}=0$. The NVT\% of the $k$ th tourist can then be 
TABLE 2: Spatial trajectories of tourists.

\begin{tabular}{lcc}
\hline Number & Spatial trajectory & $t n$ \\
\hline $\operatorname{Tr}_{1}$ & $\left\{v_{1}, v_{2}, v_{3}, v_{5}, v_{6}, v_{7}, v_{10}, v_{12}, v_{14}, v_{16}, v_{18}, v_{22}, v_{25}\right\}$ & 38 \\
$\operatorname{Tr}_{2}$ & $\left\{v_{1}, v_{4}, v_{6}, v_{7}, v_{10}, v_{11}, v_{13}, v_{15}, v_{16}, v_{20}, v_{22}, v_{25}\right\}$ & 15 \\
$\vdots$ & $\vdots$ & $\vdots$ \\
$\operatorname{Tr}_{49}$ & $\left\{v_{2}, v_{4}, v_{25}, v_{23}, v_{22}, v_{20}, v_{14}, v_{10}, v_{9}, v_{5}\right\}$ & 64 \\
$\operatorname{Tr}_{50}$ & $\left\{v_{3}, v_{4}, v_{25}, v_{23}, v_{22}, v_{21}, v_{18}, v_{16}, v_{12}, v_{10}, v_{7}, v_{5}\right\}$ & 63 \\
\hline
\end{tabular}

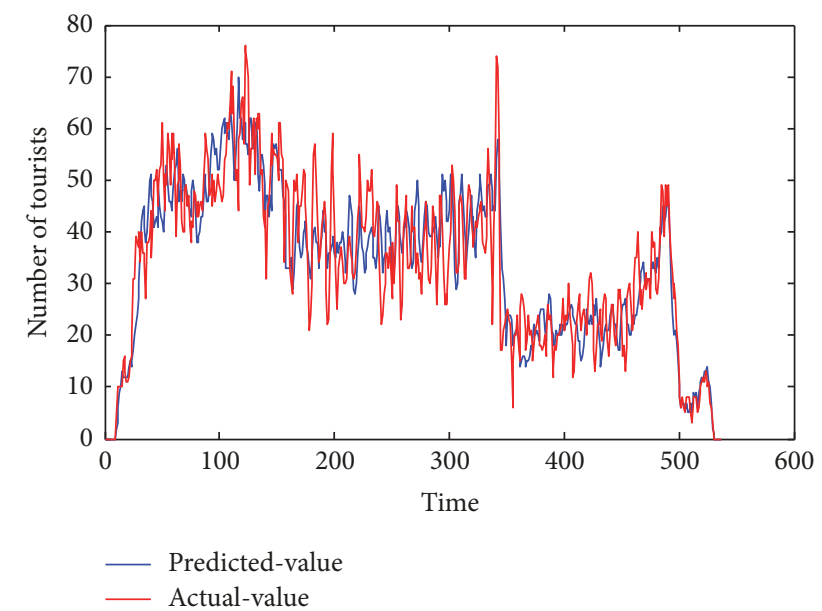

FIgURE 3: Predicted number of tourists at ride $v_{6}$.

calculated using (10), where $T_{k}$ is the total duration time in the park of the $k$ th tourist, and the average value of $\mathrm{NVT}_{k} \%$ for all of the tourists is calculated by (11):

$$
\begin{aligned}
\mathrm{NVT}_{k} \% & =\frac{\sum_{t=0}^{T} \sum_{i=1}^{N} w t_{i k}(t)+\sum_{i=1}^{N-1} \sum_{j=1}^{N} w_{i j} \cdot z_{i j k}}{T_{k}}, \\
\overline{\mathrm{NVT}} \% & =\frac{\sum_{k=1}^{\mathrm{Q}} \mathrm{NVT}_{k} \%}{\mathrm{Q}} .
\end{aligned}
$$

Coordinating tourist visit sequences step by step (also known as the single step dynamic coordination approach) has shown greater promise for crowding mitigation in theme parks than alternatives, due to the complexity and interactivity of tourist movements in a theme park [31]. Most step-by-step approaches coordinate tourist visit sequences based on the current crowd situation, while the prospective coordination approach proposed in this paper is based on predictions of tourist distributions. For the purpose of comparison, the former method, based on the current situation, is used as a baseline (baseline approach, BA). We set the tourist numbers $Q=10,000$, and all of the tourists enter the park between $8 \mathrm{a} . \mathrm{m}$. and $10 \mathrm{a} . \mathrm{m}$. The tourists' arrival distribution-the number of tourists arriving at the park every minute-follows the Poisson distribution. The tourists are numbered from 1 to 10,000 in the order of their arrival time. To reduce interference from random factors, we repeat each experiment 100 times. The NVT\% for each tourist that

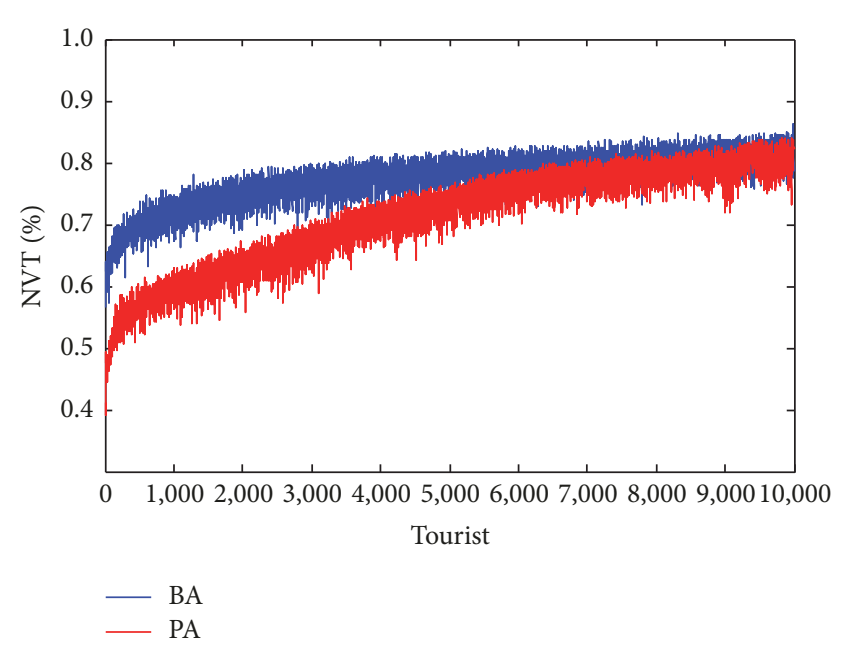

FIGURE 4: Experimental results of the two methods.

is achieved by the two approaches is presented graphically in Figure 4, where the blue lines denote the NVT\% of the baseline approach (BA), and the red lines denote our prospective approach (PA). The horizontal coordinates of Figure 4 represent the tourist ID (from 1 to 10,000), and as we can see from the figure, the later the tourists that enter the park, the more the unvalued time that they will spend, for both PA and BA. This can be explained by the fact that tourists who arrive earlier have shorter waiting times. PA outperforms BA in initial stages, although the effect weakens as the number of tourists increases.

To further analyze the performance of these two methods, an independent sample $t$-test is conducted. The means and standard deviations of $\overline{\mathrm{NVT}} \%$ for these two methods are displayed in Table 3. Table 4 shows the results of the independent samples $t$-test, where Levene's test is conducted to evaluate the equality of variances; the result $(F=24.11$, $p=0.000)$ indicates unequal variances, which means that the independent samples test results for "equal variances not assumed" should be adopted. The independent samples test results $(t(100)=294.009, p<0.05)$ indicate that our proposed approach achieves significantly smaller $\overline{\mathrm{NVT}} \%$ $(M=0.711, \mathrm{SD}=0.002)$ than the baseline approach $(M=$ $0.775, \mathrm{SD}=0.001)$. Similarly, when we further evaluate the performance of our proposed approach for a range of tourist numbers $(Q=1,000,2,000, \ldots, 15,000)$, the $t$-test results listed in Table 5 also show our proposed method performing significantly better than the baseline approach. 
TABLE 3: Group statistics $(Q=10,000)$.

\begin{tabular}{lccccr}
\hline & Method & $N$ & Mean & Std. deviation & Std. error mean \\
\hline \multirow{2}{*}{ NVT\% } & BA & 100 & .775 & 0.001 & 0.000 \\
& PA & 100 & .711 & 0.002 & 0.000 \\
\hline
\end{tabular}

TABLE 4: Independent samples test $(Q=10,000)$.

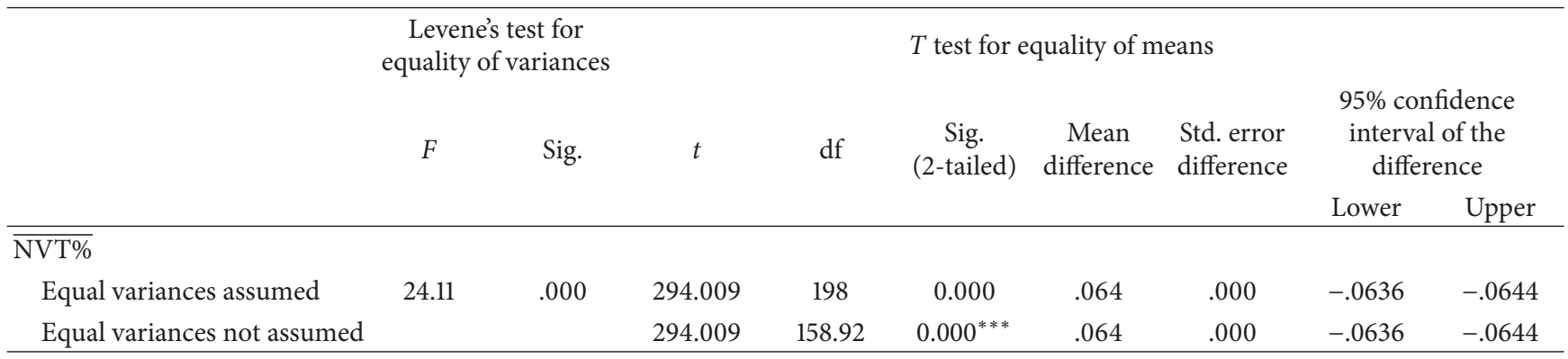

${ }^{* * *} p<0.001$.

TABLE 5: Independent samples $t$-test.

\begin{tabular}{|c|c|c|c|c|c|c|}
\hline \multirow{2}{*}{$Q$} & \multicolumn{2}{|c|}{ Mean } & \multicolumn{2}{|c|}{ SD } & \multirow{2}{*}{$t$} & \multirow{2}{*}{$p$} \\
\hline & BA & PA & BA & PA & & \\
\hline 1,000 & 0.634 & 0.445 & 0.002 & 0.001 & 410.973 & $.000^{* * *}$ \\
\hline 2,000 & 0.634 & 0.455 & 0.001 & 0.002 & 414.318 & $.000^{* * *}$ \\
\hline 3,000 & 0.634 & 0.470 & 0.001 & 0.002 & 396.840 & $.000^{* * *}$ \\
\hline 4,000 & 0.634 & 0.488 & 0.001 & 0.002 & 353.204 & $.000^{* * *}$ \\
\hline 5,000 & 0.647 & 0.524 & 0.001 & 0.003 & 175.249 & $.000^{* * *}$ \\
\hline 6,000 & 0.674 & 0.569 & 0.001 & 0.003 & 142.869 & $.000^{* * *}$ \\
\hline 7,000 & 0.704 & 0.614 & 0.001 & 0.002 & 153.155 & $.000^{* * *}$ \\
\hline 8,000 & 0.730 & 0.650 & 0.002 & 0.222 & 133.191 & $.000^{* * *}$ \\
\hline 9,000 & 0.754 & 0.682 & 0.001 & 0.002 & 119.586 & $.000^{* * *}$ \\
\hline 10,000 & 0.775 & 0.712 & 0.001 & 0.002 & 294.009 & $.000^{* * *}$ \\
\hline 11,000 & 0.793 & 0.734 & 0.001 & 0.002 & 129.638 & $.000^{* * *}$ \\
\hline 12,000 & 0.809 & 0.755 & 0.001 & 0.002 & 117.125 & $.000^{* * *}$ \\
\hline 13,000 & 0.822 & 0.773 & 0.001 & 0.002 & 107.147 & $.000^{* * *}$ \\
\hline 14,000 & 0.834 & 0.789 & 0.001 & 0.001 & 132.855 & $.000^{* * *}$ \\
\hline 15,000 & 0.845 & 0.803 & 0.001 & 0.002 & 113.732 & $.000^{* * *}$ \\
\hline
\end{tabular}

As is evident in Table 5, our proposed approach outperforms the baseline approach (BA). We further explore whether the ride parameters (e.g., carrying capacity) listed in Table 1 affect the performance of our proposed approach. First, we set tourist numbers as $Q=10,000$, with all entering the park between 8 a.m. and 10 a.m. Second, we design 30 experimental scenes, in which the carrying capacity of each ride is randomly set to the values between 50 and 500 . Third, we coordinate the tourist visit sequences for these 30 scenes using PA and BA, respectively. Finally, the paired samples $t$ test is conducted to evaluate the performance of our proposed approach. The mean and standard deviations of the $\overline{\mathrm{NVT}} \%$ achieved by both approaches are listed in Table 6, while Table 7 displays the results of the paired samples $t$-test. For the pair (PA-BA), the gap mean is -.073 . The test results indicate that PA achieves significantly smaller $\overline{\mathrm{NVT}} \%(M=$ $.695, \mathrm{SD}=.078)$ than $\mathrm{BA}(M=.768, \mathrm{SD}=.056)(t(30)=$ $-17.793, p<0.05)$, confirming the broad applicability of our approach across a range of ride parameters.

4.4. Discussion. Section 4.3 presents the tests and verifications for our prospective coordination approach. Meanwhile, in this section, we tend to design two experiments using agent-based simulation platform to explore (1) the relationships between tourists' arrival time scope and the crowding situation and (2) the relationships between the effect of coordination approaches and tourist numbers.

(1) Tourists' Arrival Time Scope and the Crowding Situation. In Section 4.3, we assume that all of the tourists enter the 
TABle 6: Paired sample statistics.

\begin{tabular}{lcccc}
\hline & Mean & $N$ & Std. deviation & Std. error mean \\
\hline PA & .695 & 30 & .078 & .014 \\
BA & .768 & 50 & .056 & .010 \\
\hline
\end{tabular}

TABle 7: Paired samples test.

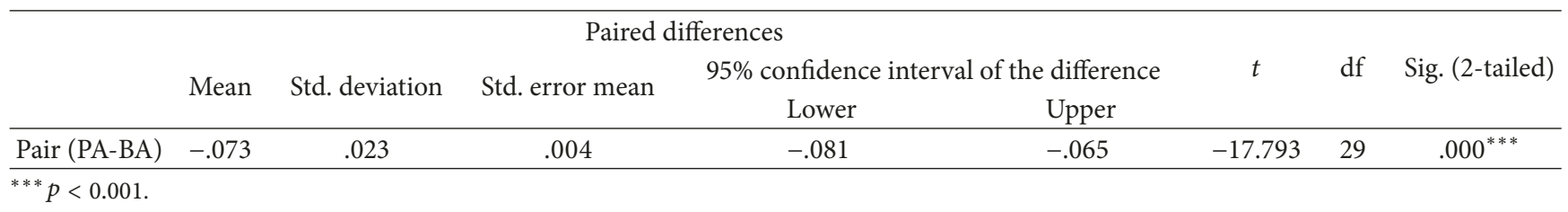

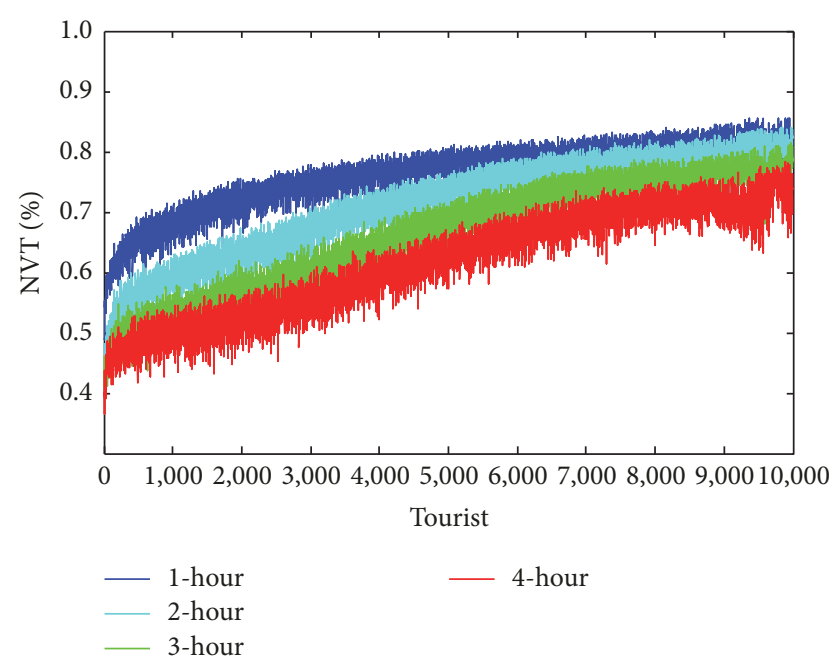

FIGURE 5: Experimental results of the four arrival time scopes.

park in a 2-hour interval (8:00 a.m. to 10:00 a.m.). In this section, we explore how the tourists' arrival time scope affects crowding by setting 1-hour (8:00 a.m. to 9:00 a.m.), 2-hour (8:00 a.m. to 10:00 a.m.), 3-hour (8:00 a.m. to 11:00 a.m.), and 4-hour (8:00 a.m. to 12:00 a.m.) entrance periods. The NVT\% for each tourist for these four entrance periods is presented graphically in Figure 5, where the dark blue lines represent the NVT\% for each tourist, when 10,000 tourists enter the park within a one-hour span (8:00 a.m. to 9:00 a.m.), while the light blue, green, and red lines denote two-hour (8:00 a.m. to 10:00 a.m.), three-hour (8:00 a.m. to 11:00 a.m.), and fourhour (8:00 a.m. to 12:00 a.m.) spans, respectively.

An analysis of variance (ANOVA) is conducted to determine the differences. The Scheffe post hoc test is carried out to identify further differences between groups if the equal variance assumption is met, and Tamhane's T2 is used if the equal variance assumption is violated. The descriptive statistics are listed in Table 8 . The results indicate that there are statistically significant differences between groups in NVT\% $(F=5977.249, p<0.05$ in Table 9). To further determine which specific groups are different, Tamhane's T2 test is conducted, as the variances of the compared groups are unequal (Levene statistic $=1733.669, p<0.05$ ) (see Table 9). The results show that when tourists enter the park
TABLE 8: Descriptive statistics.

\begin{tabular}{lccc}
\hline & $N$ & Mean & Std. deviation \\
\hline 1-hour & 10,000 & 0.7618 & 0.0551 \\
2-hour & 10,000 & 0.7107 & 0.0810 \\
3-hour & 10,000 & 0.6630 & 0.0929 \\
4-hour & 10,000 & 0.6175 & 0.0880 \\
Total & 40,000 & 0.6887 & 0.0970 \\
\hline
\end{tabular}

in a one-hour span, they experience the highest proportion of unvalued time $(M=0.7618)$; this is followed by the two-hour $(M=0.717)$, three-hour $(M=0.6630)$, and fourhour $(M=0.6175)$ spans. That is, the more dispersed the tourists' arrival, the less unvalued the time they are likely to spend. This finding suggests that some strategies could be devised for controlling variations in demand at different times. One strategy might involve revenue management, which has proven to be suitable for mitigating demand during peak hours $[3,55]$. That is, tourists entering the theme parks at different times may be charged different prices, encouraging those with high price sensitivity to choose off-peak hours. Another strategy is to require reservations, which has also been shown to be effective at controlling demand at different times. This works by setting a threshold number for each time period, and tourists need to reserve an entry slot in advance in a "first-come, first-served" manner $[3,56]$.

(2) Coordination Approaches' Effect and Tourist Numbers. We also conducted an experiment to explore the relationships between the effect of coordination approaches and tourist numbers. In addition to the BA and PA introduced in Section 4.3, another approach, a random approach (RA), is proposed for the purpose of comparison. In RA, no coordination strategy is provided; tourists select their next destination randomly. The results are shown in Figure 6, and to our surprise when the tourist numbers are less than 5,000, RA achieved significantly less $\overline{\mathrm{NVT} \%}$ than BA. This suggests that coordination approaches based on the current situation (BA) have no obvious effect. One reason may be that coordination approaches reduce the diversity of tourists' route choices, thereby increasing the possibility of tourists gathering at a select number of specific rides. In cases where tourist numbers are low, the positive effects of the 
TABLE 9: ANOVA results for different arrival time distributions.

\begin{tabular}{|c|c|c|c|c|c|c|}
\hline & Sum of squares & $\mathrm{df}$ & Mean square & $F$ & Sig. & Tamhane's T2 $(p<0.05)$ \\
\hline Between groups & 116.473 & 3 & 38.824 & $5977.249^{* * *}$ & .000 & 1-hour $>2-, 3-, 4$-hour \\
\hline Within groups & 259.788 & 39996 & 0.006 & & & 2-hour > 3-, 4-hour 3-hour \\
\hline Total & 376.260 & 39996 & & & & $>4$-hour \\
\hline
\end{tabular}

${ }^{* * *} p<0.001$.

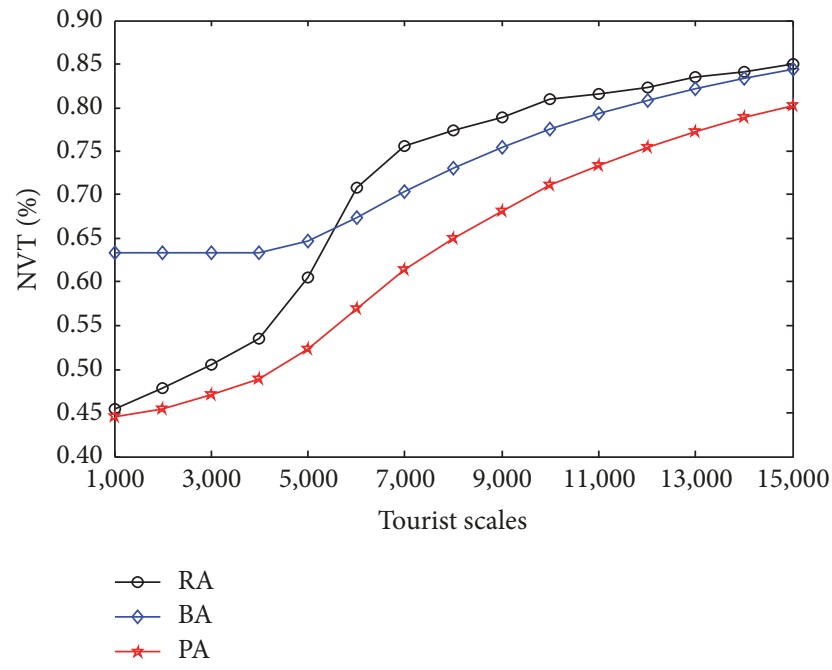

FIGURE 6: The relationships between the effect of coordination and tourist numbers.

coordination mechanism cannot compensate for its negative effects. As the number of tourists increases, the effectiveness of the coordination mechanism also increases. The situation changes again when tourist numbers exceed 11,000, where the effect of the coordination mechanism reduces, as the cause of crowding may have shifted from an uneven distribution of tourists to the overall demand exceeding absolute supply. In this case, adding new rides or limiting the number of tourists will be a more effective way to reduce tourists' unvalued time and improve visitor experiences [12].

The results of the experiment indicate the following: (1) in the case of small tourist numbers, reducing unnecessary coordination and constraints on tourists can help improve the tourist experience; (2) as the tourist numbers increase, coordination mechanisms are helpful in reducing unvalued time; and (3) when tourist numbers exceed a certain threshold, reducing the amount of tourists' unvalued time requires an increase in facilities' capacity or limiting the number of tourists overall, rather than merely coordinating tourists' visit sequences.

\section{Conclusions and Future Research}

In this study, we develop a prospective coordination approach to mitigate theme park crowding. The aim is to improve tourists' experience and make theme parks more competitive. Our research uses Markov models to develop a method for predicting tourist distribution that can improve crowding mitigation. An agent-based simulation model is constructed to validate the approach. The results indicate that the proposed method significantly outperforms existing methods. Furthermore, several management recommendations are put forward through our expanded discussion.

Our results significantly contribute to the literature and provide a number of guidelines for management. First, our study offers an improved method for crowding mitigation. Previous research focused on developing effective methods for crowding management in recreation areas has resulted in crowding oscillation due to the delay between the decision making and effect emergence [5, 26, 29-32]. Our method generally achieves better crowding mitigation by proposing a prospective coordination approach based on predictions of tourist distribution. Second, our study improves crowding management, which plays a fundamental role in improving tourists' experience and makes theme parks more competitive.

There are several possible directions for future research. First, it would be valuable to consider how tourist movement patterns and preferences affect crowding in theme parks. The coordination measures taken to mitigate crowding should result in a beneficial trade-off between tourists' personalized needs and the general interests of the parks, which is particularly important in the postmodern tourism era. Second, a more reasonable index should be designed to evaluate tourist experiences, as there are diverse kinds of waiting lines and queuing systems. Last, further exploration of agent-based simulation applications should be undertaken, including the interactions between the environment and tourists' activities, the effects of geographical accessibility on tourists' activity choice [57], and the relationships between management decisions and outcomes.

\section{Abbreviations}

V: The set of rides in the theme park

$v_{i}$ : The $i$ th ride in the theme park, $i=1,2, \ldots, N$

$w_{i j}$ : The travel time needed between each pair of rides $\left(v_{i}, v_{j}\right)$

$t_{i}: \quad$ The duration of each ride $v_{i}$

$c_{i}$ : The carrying capacity of ride $v_{i}$

$\phi_{i}(t)$ : The number of tourists at ride $v_{i}$ at moment $t$

$\delta_{i j}(t)$ : The transition probability from $v_{i}$ to $v_{j}$ at moment $t$

$\mathbf{P}(t): \quad$ The spatial-temporal transition matrix at moment $t$

$\mathrm{DV}(t)$ : The tourist distribution at moment $t$. 


\section{Conflicts of Interest}

The authors declare that they have no conflicts of interest.

\section{Acknowledgments}

This research received grants from the National Natural Science Foundation of China (no. 71601164), Humanities and Social Science Projects of the Ministry of Education (no. 16YJC630177), and Education \& Scientific Research Project of Young and Middle-Aged Teachers in Fujian Province (no. JAS160017) and was supported by the Fundamental Research Funds for the Central Universities (no. 20720161005).

\section{References}

[1] J. Ma, J. Gao, N. Scott, and P. Ding, "Customer delight from theme park experiences. The Antecedents of Delight based on Cognitive Appraisal Theory.," Annals of Tourism Research, vol. 42, pp. 359-381, 2013.

[2] B. M. Braun and M. D. Soskin, "Theme Park Competitive Strategies," Annals of Tourism Research, vol. 26, no. 2, pp. 438442, 1999.

[3] C. Y. Heo and S. Lee, "Application of revenue management practices to the theme park industry," International Journal of Hospitality Management, vol. 28, no. 3, pp. 446-453, 2009.

[4] W. H. Martin and S. Mason, "Social trends and tourism futures," Tourism Management, vol. 8, no. 2, pp. 112-114, 1987.

[5] A. Brown, J. Kappes, and J. Marks, "Mitigating Theme Park Crowding with Incentives and Information on Mobile Devices," Journal of Travel Research, vol. 52, no. 4, pp. 426-436, 2013.

[6] M. Li, B. Wu, and L. Cai, "Tourism development of World Heritage Sites in China: A geographic perspective," Tourism Management, vol. 29, no. 2, pp. 308-319, 2008.

[7] X. Cui and C. Ryan, "Perceptions of place, modernity and the impacts of tourism - Differences among rural and urban residents of Ankang, China: A likelihood ratio analysis," Tourism Management, vol. 32, no. 3, pp. 604-615, 2011.

[8] J. Alegre and J. Garau, "Tourist satisfaction and dissatisfaction," Annals of Tourism Research, vol. 37, no. 1, pp. 52-73, 2010.

[9] Y. Zhang, X. Li, Q. Su, and X. Hu, "Exploring a theme park's tourism carrying capacity: A demand-side analysis," Tourism Management, vol. 59, pp. 564-578, 2017.

[10] G. W. McClung, "Theme park selection. Factors influencing attendance," Tourism Management, vol. 12, no. 2, pp. 132-140, 1991.

[11] Q. Jin and P. Pearce, "Tourist perception of crowding and management approaches at tourism sites in Xian," Asia Pacific Journal of Tourism Research, vol. 16, no. 3, pp. 325-338, 2011.

[12] A. Kemperman, A. Borgers, H. Oppewal, and H. Timmermans, "Predicting the duration of theme park visitors' activities: An ordered logit model using conjoint choice data," Journal of Travel Research, vol. 41, no. 4, pp. 375-384, 2003.

[13] C.-Y. Tsai and S.-H. Chung, "A personalized route recommendation service for theme parks using RFID information and tourist behavior," Decision Support Systems, vol. 52, no. 2, pp. 514-527, 2012.

[14] H. Briassoulis, "Sustainable tourism and the question of the commons," Annals of Tourism Research, vol. 29, no. 4, pp. 10651085, 2002.
[15] H. Kawamura, T. Kataoka, A. Ohuchi, and K. Kurumatani, "Investigation of Global Performance Affected by Congestion Avoiding Behavior in Theme Park Problem," IEEJ Transactions on Electronics, Information and Systems, vol. 124, no. 10, pp. 1922-1929, 2004.

[16] T. Kataoka, H. Kawamura, K. Kurumatani, and A. Ohuchi, "Distributed Visitors Coordination System in Theme Park Problem," in Massively Multi-Agent Systems I, vol. 3446 of Lecture Notes in Computer Science, pp. 335-348, Springer Berlin Heidelberg, Berlin, Heidelberg, 2005.

[17] C. M. Rump and S. Stidham Jr., "Stability and chaos in input pricing for a service facility with adaptive customer response to congestion," Management Science, vol. 44, no. 2, pp. 246-261, 1998.

[18] Y. Ikeda and S. Tokinaga, "Controlling the chaotic dynamics by using approximated system equations obtained by the genetic programming," IEICE Transactions on Fundamentals of Electronics, Communications and Computer Sciences, vol. E84A, no. 9, pp. 2118-2127, 2001.

[19] P. Jakus and W. D. Shaw, "Congestion at recreation areas: Empirical evidence on perceptions, mitigating behaviour and management preferences," Journal of Environmental Management, vol. 50, no. 4, pp. 389-401, 1997.

[20] H. Lee and A. R. Graefe, "Crowding at an arts festival: Extending crowding models to the frontcountry," Tourism Management, vol. 24, no. 1, pp. 1-11, 2003.

[21] B. Neuts and P. Nijkamp, "Tourist crowding perception and acceptability in cities. An Applied Modelling Study on Bruges," Annals of Tourism Research, vol. 39, no. 4, pp. 2133-2153, 2012.

[22] A. Zehrer and F. Raich, "The impact of perceived crowding on customer satisfaction," Journal of Hospitality and Tourism Management, vol. 29, pp. 88-98, 2016.

[23] A. R. Graefe, J. J. Vaske, and F. R. Kuss, "Social carrying capacity: An integration and synthesis of twenty years of research," Leisure Sciences, vol. 6, no. 4, pp. 395-431, 1984.

[24] M. D. Needham, R. B. Rollins, and C. J. Wood, "Site-specific encounters, norms and crowding of summer visitors at alpine ski areas," International Journal of Tourism Research, vol. 6, no. 6, pp. 421-437, 2004.

[25] R. M. W. Rathnayake, "How does 'crowding' affect visitor satisfaction at the Horton Plains National Park in Sri Lanka?" Tourism Management Perspectives, vol. 16, pp. 129-138, 2015.

[26] R. H. Ahmadi, "Managing capacity and flow at theme parks," Operations Research, vol. 45, no. 1, pp. 1-13, 1997.

[27] M. Berrada and K. E. Stecke, "Branch and bound approach for machine load balancing in flexible manufacturing systems," Management Science, vol. 32, no. 10, pp. 1316-1335, 1986.

[28] F. Hamhoum and C. Kray, "Supporting pilgrims in navigating densely crowded religious sites," Personal and Ubiquitous Computing, vol. 16, no. 8, pp. 1013-1023, 2012.

[29] P. Ge et al., "Simulation study based on the regional space-time load balancing of Jiuzhaigou," Journal of Industrial Engineering and Engineering Management, vol. 27, no. 2, pp. 115-124, 2013.

[30] W. M. Zheng, M. Z. Jin, and P. Y. Ren, "The impact of information sharing on congestion using agent-based simulation," International Journal of Simulation Modelling, vol. 13, no. 2, pp. 183-194, 2014.

[31] J. Li and D. Zhu, "Multi-stage dynamic coordination and control model for mass crowds activities based on multi-agent," Chinese Journal of Management Science, vol. 17, no. 5, pp. 113-119, 2009 (Chinese). 
[32] J. Li et al., "Visitors coordination for World Expo Shanghai," Systems Engineering, vol. 6, pp. 50-56, 2010.

[33] M. Alexander, A. MacLaren, K. O'Gorman, and C. White, "Priority queues: Where social justice and equity collide," Tourism Management, vol. 33, no. 4, pp. 875-884, 2012.

[34] C.-H. Wang, "Predicting tourism demand using fuzzy time series and hybrid grey theory," Tourism Management, vol. 25, no. 3, pp. 367-374, 2004.

[35] S. F. Witt and C. A. Witt, "Forecasting tourism demand: A review of empirical research," International Journal of Forecasting, vol. 11, no. 3, pp. 447-475, 1995.

[36] C. Lim, "Review of international tourism demand models," Annals of Tourism Research, vol. 24, no. 4, pp. 835-849, 1997.

[37] G. Li, H. Song, and S. F. Witt, "Recent developments in econometric modeling and forecasting," Journal of Travel Research, vol. 44, no. 1, pp. 82-99, 2005.

[38] H. Song and G. Li, "Tourism demand modelling and forecasting-a review of recent research," Tourism Management, vol. 29, no. 2, pp. 203-220, 2008.

[39] S. I. Stewart and C. A. Vogt, "Multi-Destination Trip Patterns," Annals of Tourism Research, vol. 24, no. 2, pp. 458-461, 1997.

[40] J. C. Hallo, J. A. Beeco, C. Goetcheus, J. McGee, N. G. McGehee, and W. C. Norman, "GPS as a Method for Assessing Spatial and Temporal Use Distributions of Nature-Based Tourists," Journal of Travel Research, vol. 51, no. 5, pp. 591-606, 2012.

[41] Y. Zhang, J.-H. Xu, and P.-J. Zhuang, "The spatial relationship of tourist distribution in Chinese cities," Tourism Geographies, vol. 13, no. 1, pp. 75-90, 2011.

[42] Y. Yang and K. K. F. Wong, "Spatial Distribution of Tourist Flows to China’s Cities," Tourism Geographies, vol. 15, no. 2, pp. 338363, 2013.

[43] X. Yang and Q. Wang, "Exploratory space-time analysis of inbound tourism flows to China cities," International Journal of Tourism Research, vol. 16, no. 3, pp. 303-312, 2014.

[44] N. Leiper, "Tourist attraction systems," Annals of Tourism Research, vol. 17, no. 3, pp. 367-384, 1990.

[45] W. Zheng, X. Huang, and Y. Li, "Understanding the tourist mobility using GPS: Where is the next place?" Tourism Management, vol. 59, pp. 267-280, 2017.

[46] X. Huang and B. Wu, "Intra-attraction Tourist Spatial-Temporal Behaviour Patterns," Tourism Geographies, vol. 14, no. 4, pp. 625-645, 2012.

[47] P. Ren, Z. Liao, and P. Ge, "Real-time forecast of tourists distribution based on the improved k-means method," Mathematical Problems in Engineering, vol. 2014, Article ID 457197, 2014.

[48] J. Xia, P. Zeephongsekul, and D. Packer, "Spatial and temporal modelling of tourist movements using Semi-Markov processes," Tourism Management, vol. 32, no. 4, pp. 844-851, 2011.

[49] P. A. Johnson and R. E. Sieber, "An individual-based approach to modeling tourism dynamics," Tourism Analysis, vol. 15, no. 5, pp. 517-530, 2010.

[50] S. R. Lawson, "Computer simulation as a tool for planning and management of visitor use in protected natural areas," Journal of Sustainable Tourism, vol. 14, no. 6, pp. 600-617, 2006.

[51] K. Moore, C. Smallman, J. Wilson, and D. Simmons, "Dynamic in-destination decision-making: An adjustment model," Tourism Management, vol. 33, no. 3, pp. 635-645, 2012.

[52] A. H. Underhill, A. B. Xaba, and R. E. Borkan, "The wilderness use simulation model applied to Colorado River boating in Grand Canyon National Park, USA," Journal of Environmental Management, vol. 10, no. 3, pp. 367-374, 1986.
[53] A. Lew and B. McKercher, "Modeling tourist movements: a local destination analysis," Annals of Tourism Research, vol. 33, no. 2, pp. 403-423, 2006.

[54] J. H. Ahrens and U. Dieter, "Computer methods for sampling from gamma, beta, poisson and bionomial distributions," Computing, vol. 12, no. 3, pp. 223-246, 1974.

[55] Z. Schwartz, W. Stewart, and E. A. Backlund, "Visitation at capacity-constrained tourism destinations: exploring revenue management at a national park," Tourism Management, vol. 33, no. 3, pp. 500-508, 2012.

[56] A. Milman, "The future of the theme park and attraction industry: A management perspective," Journal of Travel Research, vol. 40, no. 2, pp. 139-147, 2001.

[57] A. Pramono and H. Oppewal, "Accessibility and the role of the consideration set in spatial choice modelling: A simulation study," Journal of Choice Modelling, vol. 5, no. 1, pp. 46-63, 2012. 


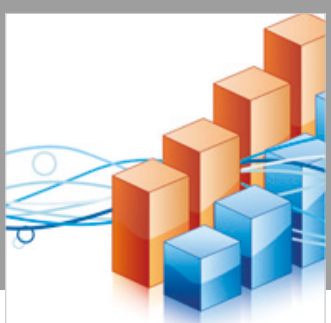

Advances in

Operations Research

\section{-n-m}
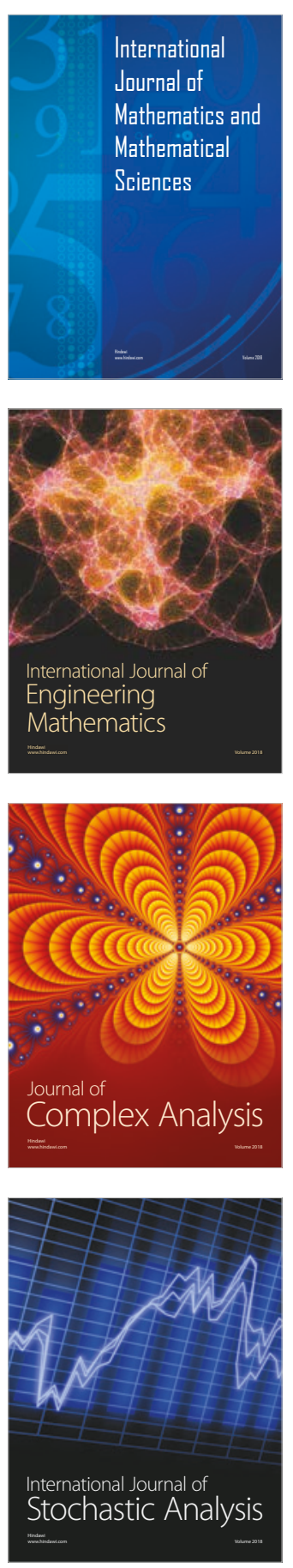
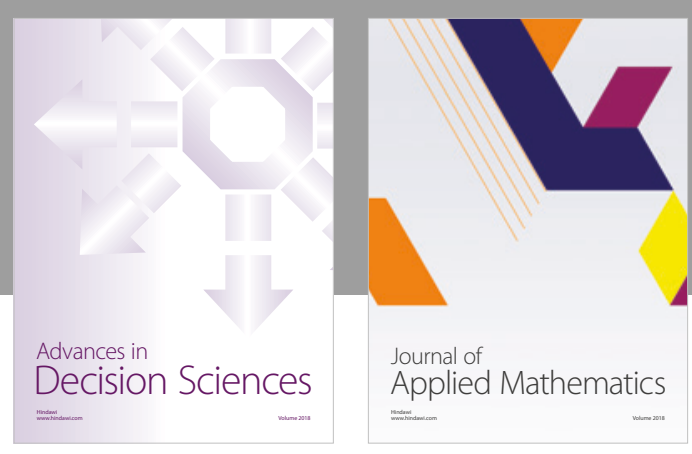

Journal of

Applied Mathematics
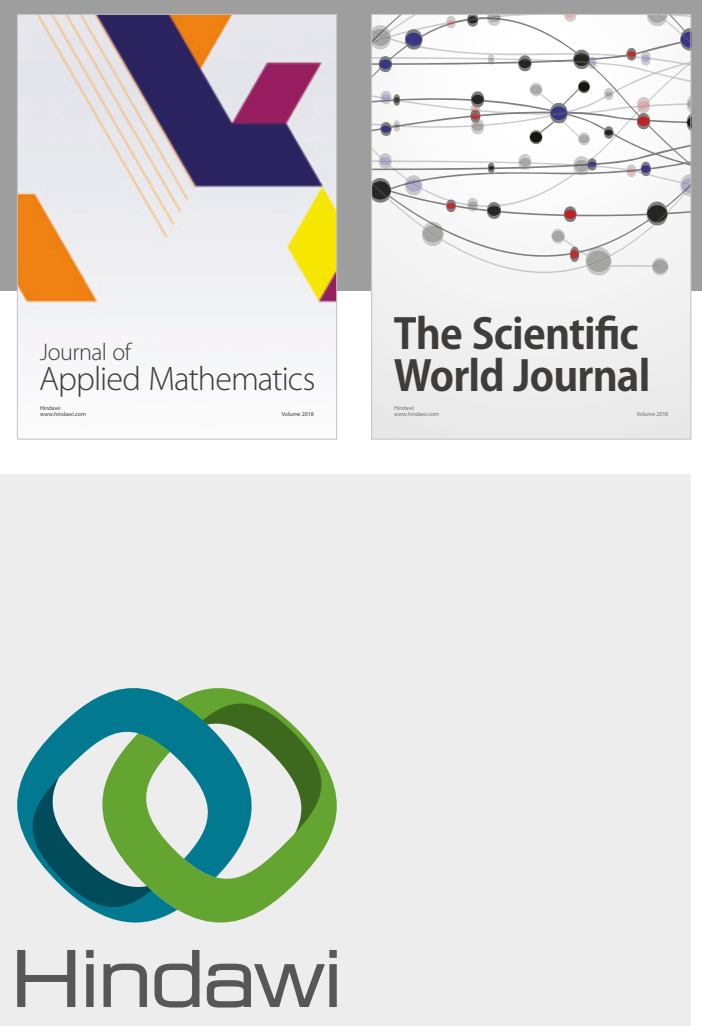

Submit your manuscripts at

www.hindawi.com

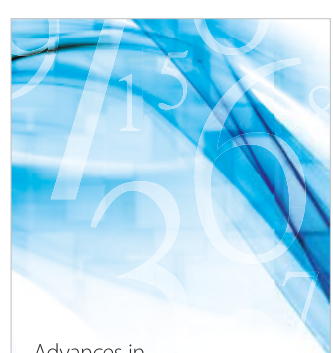

Advances in
Numerical Analysis
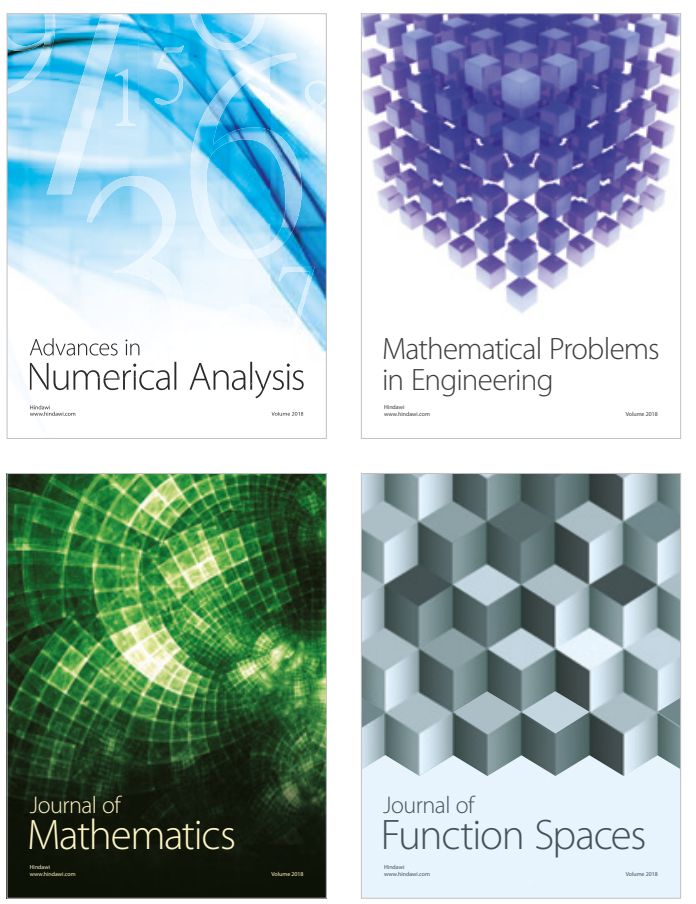

Mathematical Problems in Engineering

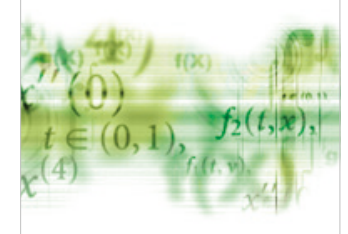

International Journal of

Differential Equations

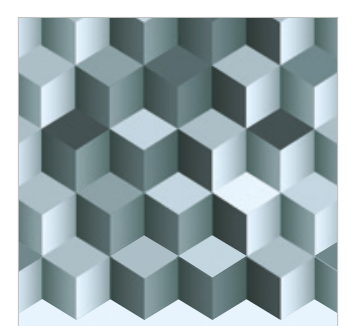

Journal of

Function Spaces

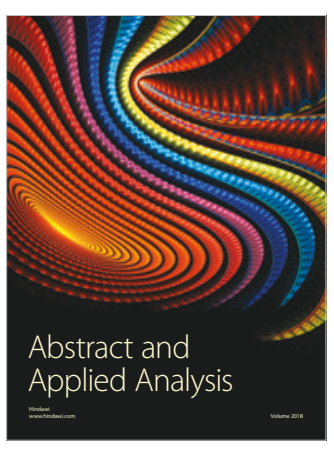

The Scientific

World Journal

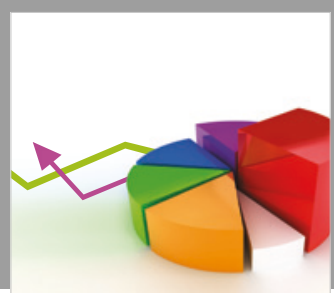

Journal of

Probability and Statistics
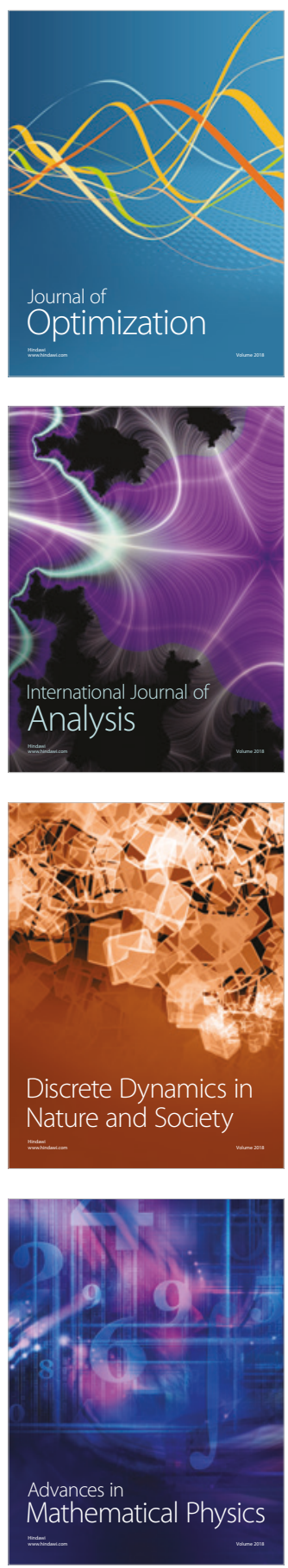